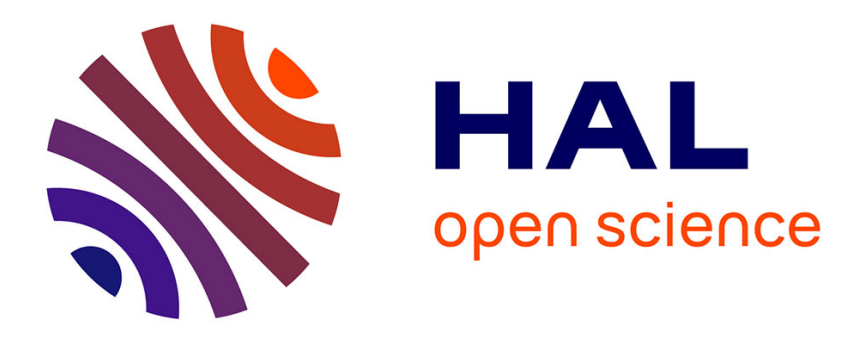

\title{
Anomalous viscous retardation of a mechanical wave at percolation threshold
}

\author{
S. Roux, A. Hansen
}

\section{To cite this version:}

S. Roux, A. Hansen. Anomalous viscous retardation of a mechanical wave at percolation threshold. Journal de Physique, 1988, 49 (6), pp.897-900. 10.1051/jphys:01988004906089700 . jpa-00210777

\section{HAL Id: jpa-00210777 https://hal.science/jpa-00210777}

Submitted on 1 Jan 1988

HAL is a multi-disciplinary open access archive for the deposit and dissemination of scientific research documents, whether they are published or not. The documents may come from teaching and research institutions in France or abroad, or from public or private research centers.
L'archive ouverte pluridisciplinaire HAL, est destinée au dépôt et à la diffusion de documents scientifiques de niveau recherche, publiés ou non, émanant des établissements d'enseignement et de recherche français ou étrangers, des laboratoires publics ou privés. 


\title{
Anomalous viscous retardation of a mechanical wave at percola- tion threshold
}

\author{
S. Roux $\left({ }^{1}\right)$ and A. Hansen $\left({ }^{2}\right)$
}

(1) Laboratoire de Physique de la Matière Hétérogène, Unité Associée au CNRS 857, Ecole Supérieure de Physique et Chimie Indu'trielles de Paris, 10 rue Vauquelin, 75231 Paris Cedex 05, France

(2) Groupe de Physique des Solides, Ecole Normale Supérieure, 24 rue Lhomond, 75231 Paris Cedex 05 , France

(Reçu le 18 février 1988, accepté le 90 mars 1988)

Résumé.- Nous étudions le temps moyen nécessaire à la propagation d'un choc mécanique à travers un treillis réticulé aléatoirement dégradé dans un milieu visqueux, au seuil de percolation de forces centrales. Ce problème est l'analogue mécanique du phénomène de diffusion anormale. Nous montrons, par une simulation numérique de matrice de transfert, que le temps moyen $<t>$ de propagation d'une impulsion sur un réseau de taille $L$ varie selon $\left\langle t>\propto L^{2+\theta^{\prime}}\right.$.

\begin{abstract}
We study the mean transit time needed for a mechanical shock to propagate through a random depleted lattice of freely-rotating elastic springs in a viscous medium at the central-force percolation threshold. This problem is the exact mechanical counterpart of anomalous diffusion. We show that the mean transit time $\langle t\rangle$ for a pulse to cross a lattice of size $L$, scales as $\langle t\rangle \propto$ $L^{2+\theta^{\prime}}$ through a transfer-matrix analysis.
\end{abstract}

\section{Introduction.}

Two different models have been considered in the framework of mechanical systems at the percolation threshold. The first one introduced by Kantor and Webman [1] which includes angular elasticity, is now well understood as far as static properties are concerned (i.e. either rigidity vanishing at percolation threshold [2] or rigidity divergence for the super-elastic problem [3] ). The second one, the central-force model, introduced by Feng and Sen [4], does not include any angular elasticity term. The latter is by far more controversial in the litterature $[4-9]$.
Formally, vector-transport properties (e.g. elasticity) are very close to scalar transport properties (e.g. conductivity) and most features of the scalar transport have their natural counterpart in a mechanical problem. Thus, it is not surprising to find a mechanical analog of anomalous diffusion [10] . The latter phenomenon arises from the study of the diffusion of random walkers on a self-similar geometry such as a randomly depleted lattice right at the percolation threshold. It leads to a hypodiffusive behaviour : the mean time $<t>$ needed for a random walker to cross a distance $R$ scales as $<t>\propto R^{2+\theta}$ where $\theta$ is related to other $\mathrm{dy}-$ 
namic and static critical exponents. In the following, we will be concerned with an analogous property that will be studied in the framework of central-force percolation. However, a similar phenomenon should also occur for systems with angular elasticity near the percolation threshold.

\section{Model.}

Let us consider an elastic spring in a viscous medium such that the displacement $U(x, t)$ along the spring at a time $t$ and at a distance $x$ from one end varies according to

$$
\partial U(x, t) / \partial t=K \partial^{2} U(x, t) / \partial x^{2}
$$

$K$ is a visco-elastic constant set to the value 1 in the following. (This equation is to be compared with one dimensional diffusion equation where the quantity $U$ would then be a concentration). We consider now a triangular lattice made out of such springs, and remove at random a fraction $(1-p)$ of these bonds.

We know that for $p$ below the central-force percolation threshold, (here for a triangular lattice $p_{\mathrm{c}} \approx 0.642[8,9]$ ) the system is no longer rigid and, therefore, if we push on one side of the lattice, the impulse will not propagate further than the correlation length $\xi$. On the other hand when $p$ is larger than $p_{\mathrm{c}}$, the system is rigid and the signal will propagate. However this propagation will take some time. It is this time delay whose critical behaviour we study here.

\section{Method.}

The method we utilized to study this problem is very similar in spirit to the one used to analyse anomalous diffusion $[11,12]$. The first step is to Laplace transform in time equation (1)

$$
s \mathbf{U}(x, s)+\partial^{2} \mathbf{U}(x, s) / \partial x^{2}=0
$$

(where we made the choice $K=1$ ). This equation can be solved directly, and we can write the forces $\mathbf{F}=\partial \mathbf{U} / \partial x$ at each end (say 1 and 2) of one bond as a linear function of the displacements at the same points.

$$
\left[\begin{array}{l}
F_{1} \\
F_{2}
\end{array}\right]=\left[\begin{array}{ll}
\alpha & \beta \\
\beta & \alpha
\end{array}\right] \cdot\left[\begin{array}{l}
U_{1} \\
U_{2}
\end{array}\right]
$$

where $\alpha=\sqrt{s} \cot (l \sqrt{s})$ and $\beta=$ $-\sqrt{s} / \sin (l \sqrt{s})$ and $l$ is the length of one bond. For now on, we will set $l=1$. Now, $\alpha, \beta$ and $F$ are complicated functions of $s$. We develop every quantity introduced so far in series of $s$ up to the first order (and label with superscripts the different orders in $s$ ).

$$
\mathbf{F}=\mathbf{F}^{0}+\mathbf{F}^{1} s+O\left(s^{2}\right)
$$

We can then express

$$
\left[\begin{array}{l}
F_{1}^{0} \\
F_{2}^{0}
\end{array}\right]=\left[\begin{array}{cc}
1 & -1 \\
-1 & 1
\end{array}\right]\left[\begin{array}{l}
U_{1}^{0} \\
U_{2}^{0}
\end{array}\right]
$$

and

$$
\left[\begin{array}{l}
F_{1}^{1} \\
F_{2}^{1}
\end{array}\right]=\left[\begin{array}{cc}
1 & -1 \\
-1 & 1
\end{array}\right] \cdot\left[\begin{array}{l}
U_{1}^{1} \\
U_{2}^{1}
\end{array}\right]-1 / 6\left[\begin{array}{ll}
2 & 1 \\
1 & 2
\end{array}\right] \cdot\left[\begin{array}{l}
U_{1}^{0} \\
U_{2}^{0}
\end{array}\right]
$$

In order to describe the behaviour of the whole lattice, we have to specify an additional rule; namely, the balance of forces at each node, to all orders in $s$. All the computation is now performed by treating each order in $s$ independently.

\section{Algorithm.}

We will study the critical behaviour by a transfer matrix algorithm. We build a long strip (length $\approx 10^{5}$ ) of small width (from 2 to 20 ) of a triangular lattice right at the percolation threshold, $p_{c}=0.642$, as determined in previous works $[8,9]$. The spirit of the method consists in computing the matrix that relates the forces at each site of the end of the strip to the displacement at the same set of sites. This matrix is updated every time we add a single bond to the strip. As we have noted previously, we treat separately the first two orders in the $s$ expansion of all quantities. In fact we are dealing with two matrices, as can be seen from equation (5) : the first one relates the zeroth order of forces to the zeroth order of displacements. The same matrix gives the dependence of the first order of forces to the first order of displacements. The second matrix provides the additional dependence of the first order of forces on the zeroth order of displacements. Both of these matrices are symmetric.

The boundary conditions are the following: along the axis of the strip, both edges are 
connected to two rigid bars. One of these bars is fixed whereas the other one is free. On the latter, we impose a unit pulse $F(t)=\delta(t)$ (a Dirac distribution) either along the strip -shearor transverse to it -compression- at time $t=0$. If one would record the force exerted by the lattice onto the fixed bar as a function of time, then the result would be a signal $\phi(t)$ such that :

$$
\int \phi(t) \mathrm{d} t=\int F(t) \mathrm{d} t=1
$$

This equation expresses the fact that the whole impulse reaches the fixed bar (in the same way as in the electrical analog where it expresses the charge conservation). We will concentrate hereafter on the mean time $\langle t\rangle$ needed by the pulse to cross the strip :

$$
<t>=\int t \phi(t) \mathrm{d}(t)
$$

In practice, we compute the Laplace transform in time $\phi(s)$ of the signal $\phi(t)$. If we expand in powers of $s$, we obtain using equations (6) and (7) :

$$
\phi(s)=1-<t>s+O\left(s^{2}\right)
$$

Since in our computation we treat each order in $s$ independently, we obtain directly the result $<t>$. Obviously this kind of treatment can be done up to any order $n$. It would just require some additionnal matrices ( $n$ matrices are needed to study up to the $(n-1)$ th order. If one would have been interested in real-time response, then dealing with only one matrix but for different values of the parameter $s$, would have been enough for an inverse Laplace transform to be performed on the result so as to get the real time response.

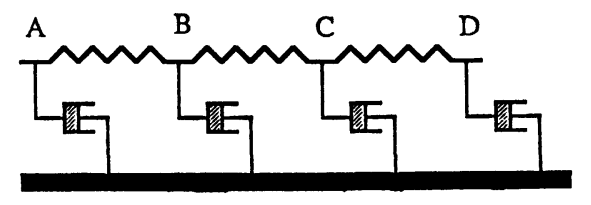

Fig. 1.- In this work, we consider visco-elastic elements schematically represented on this figure. Each sites of the lattice $(\mathrm{A}, \mathrm{B}, \mathrm{C} . .$.$) is$ connected to some of its neighbours (with probability $p$ ) by springs, and to the ground (always) by a viscous damper. We study the diffusive propagation of a shock through this system.

JOURNAL DE PHYSIQUE. - T. 49, N 6, JUIN 1988
Results.

Our algorithm provides the value of the mean transit time for a pulse to propagate throughout the strip of width $w$. In an homogeneous lattice, we have the following dependence

$$
<t>\propto w^{2}
$$

which reflect the diffusive nature of the propagation.

At percolation threshold, in analogy with the scalar case, we expect an anomalous powerlaw regime :

$$
<t>\propto w^{2+\theta^{\prime}}
$$

Figure 2 displays the results obtained : for small width, the slope of the curve shown (which should be $2+\theta^{\prime}$ if the scaling Eq. (9) were valid) is smaller than for larger width : this indicates that corrections to scaling are important in our case, as it has already been shown to be of the uttermost relevance in other studies of static mechanical properties $[2,8]$. For the largest width, we can extract from the data shown an estimate of the apparent slope : $2+\theta^{\prime}=5.0 \pm 0.5$. This result is to be compared with the scalar case where the difference between the percolation regime and the well-connected one is much less important : $\left\langle t>\propto w^{2+\theta}\right.$ where $\theta=0.87$ in the two dimensional case.

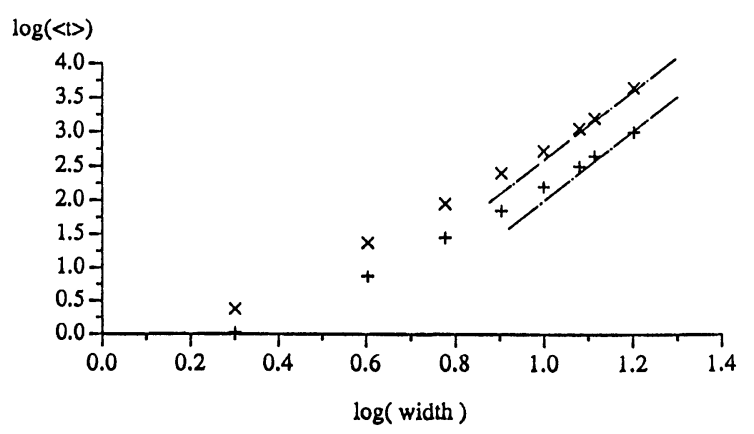

Fig. 2.- Log-log plot of the mean transit time needed for the impulse to cross a strip of width $L$, versus the width $L$. The upper curve refers to shear, and the lower to compression. The slope of these curves should tend to the value $2+\theta$, introduced in the text. (A slope of 5 is shown for comparison).

In the latter case, we know that $\theta=(t-$ $\beta) / \nu$ where $t$ is the critical exponent of the conductivity, $\beta$ is the one of the probability for a site to belong to the infinite cluster, and $\nu$ is 
the correlation length exponent. If we transpose this formula to the case of central-force elasticity then, using the estimate $t / \nu=3.1 \pm$ $0.1[5,7]$ and assuming that $\beta / \nu$ (here the fractal codimension of the infinite cluster of rigid sites) is close to its value in usual percolation problem (i.e. $\beta / \nu=0.1$ ), we obtain the estimate $2+\theta^{\prime}=5.0 \pm 0.1$ in agreement with our result.

These properties can also be related to the vibrational spectrum of elastic lattices with masses at each node. The density of states $n(\omega)$ should scale as $w^{d_{s}-1}$ where $d_{\mathrm{s}}=2 d_{\mathrm{f}} /(2+$ $\left.\theta^{\prime}\right)[10]$. Assuming once again that the fractal dimension of the infinite rigid cluster $d_{\mathrm{f}}$ for the central-force percolation case is identical to that of usual percolation yields $d_{\mathrm{s}}=0.625$ [13] obtained by small scale simulations.

\section{Conclusion.}

We introduced a novel physical phenomenon, related to a dynamical mechanical property which gives indirectly some information on a purely geometric property (namely the fractal dimension of the incipient infinite rigid cluster) at the central-force percolation threshold.
This property should be possible to observe experimentally, for instance at the sol-gel transition, and associated with static measurements it could give some indication about the geometrical nature of the rigid infinite cluster through dependence of $\theta^{\prime}$ on $\beta / \nu$.

Finally, let us stress the point that, although the present study has been devoted to central-force percolation, it should also apply to the mechanical behaviour of systems having angular elasticity at the percolation threshold. It is worth noting that in the latter case, one expects a value of $2+\theta^{\prime}=4.87$, indicating a drastic slowing down as one approaches the percolation threshold.

\section{Aknowledgements.}

We wish to acknowledge the support of the Ecole Nationale des Ponts et Chaussées (S.R.) and the C.E.N.-Saclay through Joliot Curie Fellowship (A.H.). The computations have been performed on a FPS164 supported, by the Greco Experimentation Numérique.

\section{References}

[1] Kantor, Y. and Webman, I., Phys. Rev. Lett. 52 (1984) 1891.

[2] ZABolitzky, J.G., BergmaN, D. and StAuffer, D., J. Stat. Phys. 44 (1986) 211.

[3] Bergman, D.J., Phys. Rev. B 33 (1986) 2013.

[4] Feng, S. and Sen, P.N., Phys. Rev. Lett. 52 (1984) 216.

[5] LemieuX, M.A., BretoN, P. and Tremblay, A.-M.S., J. Phys. Lett. France 46 (1985) L-1;

DAY, A.R., TREMBLAY, R.R. and TrEmblay, A.-M.S., Phys. Rev. Lett. 56 (1986) 2501 ;

SAHIMINI, M. and Goddard, J.D., Phys. Rev. B 32 (1985) 1869.
[6] Garcia-Molina, R., Guinea, F. and LouIS, E., Phys. Rev. Lett. 60 (1988) 124.

[7] Burton, D. and Lambert, L., Critical dynamics of a superelastic network (preprint).

[8] HANSEN, A. and RouX, S., Universality class of central-force percolation (preprint).

[9] RouX, S. and HANSEN, A., Transfer-matrix study of the elastic properties of centralforce percolation (preprint).

[10] Gefen, Y., Aharony, A., Alexander, S., Phys. Rev. Lett. 50 (1983) 77.

[11] Roux, S., Mitescu, C.D., Charlais, E. and BAUdet, C., J. Phys. A 19 (1986) L687.

[12] Roux, S. and GUYON, E. Europhys. Lett. 4 (1987) 175.

[13] LAM, P.M. and BAO, W., Z. Phys. B 59 (1985) 333. 